\title{
TERMORREGULACIÓN DE LAGARTOS EN LA FORMACIÓN DE PROFESORES DE CIENCIAS NATURALES Y EDUCACIÓN AMBIENTAL
}

\section{Thermoregulation of lizards in the training of teachers of natural sciences and environmental education}

\author{
Erika Patricia Daza-Pérez ${ }^{1}$ \\ Royman Pérez-Miranda ${ }^{2}$
}

\begin{abstract}
Resumen: Se estudiaron las propuestas científicas sobre termorregulación en lagartos para integrar un modelo a partir del cual, mediante un cuestionario y análisis de contenido, se analizaron las explicaciones sobre el fenómeno presentadas por profesores de ciencias en formación pertenecientes a un programa de Licenciatura en Ciencias Naturales. Los modelos de los profesores distan del modelo científico. Con base en ello se presenta una propuesta y se argumenta sobre la importancia de hacer objeto de trabajo dicha temática en la formación de esos profesores.
\end{abstract}

Palabras clave: Formación de profesores. Termorregulación. Lagartos. Modelos.

\begin{abstract}
We studied the scientific proposals on thermoregulation in lizards to build a model from which, through a questionnaire and content analysis, we analyzed the explanations of the phenomenon presented by science teachers training. The models of the professors are far from scientific model. On this basis we present a proposal and argues for the importance of working to that theme in the training of these teachers.
\end{abstract}

Keywords: Training teachers. Thermoregulation. Lizards. Models.

\footnotetext{
${ }^{1}$ Magíster en Docencia de la Química. Coordinadora académica - Instituto Agrícola de Alto Jordán, Vélez Santander Colombia e Investigadora Grupo GECOS Universidad Pedagógica y Tecnológica de Colombia. Tunja, Boyacá, Colombia. <erdaza1212@yahoo.es>

${ }^{2}$ Magíster en Docencia de la Química. Profesor catedrático e investigador, Grupo IREC, Universidad Pedagógica. <royman@pedagogica.edu.co>
}

${ }^{1}$ Calle 23 A N. $11-04$

Málaga Santander, Colombia 


\section{Introducción}

La formación inicial de profesores de ciencias como campo de investigación ha permitido documentar ampliamente sobre las ideas de los docentes en relación con los contenidos científicos, la historia y la epistemología de la ciencia (MELLADO, 1996) o el proceso enseñanza - aprendizaje de la misma (BELTRÁN VILLAMIZAR; QUIJANO HERNÁNDEZ; VILLAMIZAR ACEVEDO, 2008) ha sido escasa la atención a aspectos relacionados con los contenidos curriculares, a la forma como estos son orientados y a la formulación y evaluación de estrategias para hacerlos objeto de trabajo en el aula.

En Colombia, desde el Ministerio de Educación Nacional (MEN), se ha enmarcado el trabajo de formación en ciencias desde la perspectiva Ciencias Naturales y Educación Ambiental. Por tanto, la estructuración de un currículo que responda a esta propuesta de formación ha de garantizar un dominio conceptual y metodológico de las ciencias de la Naturaleza (GONZÁLEZ et al., 1996; ADÚRIZ-BRAVO; IZQUIERDO; ESTANY, 2002) y la comprensión de los organismos vivos como sistemas complejos y ordenados con capacidad de autorregulación (MAYR, 2004) pues esta propiedad es considerada como uno de los conceptos dentro de los que se interpretan la mayoría de los datos fisiológicos (ECKERT et al., 2001).

Esos profesores responsables de la enseñanza de las Ciencias de la Naturaleza en la educación básica, media y tal vez universitaria, propiciarán los espacios para que sus estudiantes comparen mecanismos de obtención de energía en los seres vivos, establezcan relaciones entre el clima y las adaptaciones de los seres vivos y propongan ejemplos de principios termodinámicos en diversos sistemas (COLOMBIA, 2004).

En consecuencia, un análisis de las explicaciones que elaboran esos profesores en formación inicial sobre los fenómenos de la naturaleza, particularmente lo relacionado con la termorregulación en lagartos, daría información básica para orientar una formación que responda al enfoque propuesto.

Por estas razones, se propuso explorar los modelos que elaboran los profesores de Ciencias Naturales y Educación Ambiental de la Universidad Pedagógica y Tecnológica de Colombia (UPTC) sobre la termorregulación en lagartos, conocer hasta dónde utilizan los conocimientos básicos de física, de química y de biología para elaborar tales modelos, y a partir de ello, plantear una propuesta que permita el diseño y desarrollo de estrategias didácticas que propicien en esos profesores acudir a áreas de conocimiento como la química, la física, la matemática para elaborar modelos explicativos que den cuenta de fenómenos como el que constituye la termorregulación en lagartos.

A partir de lo propuesto por la comunidad de especialistas, el artículo explicita de forma básica el proceso de termorregulación en lagartos. A partir de ello analiza las explicaciones de los profesores y presenta una propuesta teórica para que desde este tema particular se aborden temas de física y química fundamentales en la formación de los licenciados en mención. 
Termorregulación de lagartos en la formación de profesores ...

\section{¿Cómo termorregulan los lagartos? Una síntesis}

En este y los siguientes apartados nos referiremos a la termorregulación como el proceso mediante el cual los animales regulan su temperatura corporal, y a la temperatura, como una medida del promedio de la energía cinética de un sistema térmico.

Los sistemas físicos suelen estar en el equilibrio o suficientemente cerca de él de forma que pueden ser estudiados mediante la termodinámica clásica. En su segundo principio, afirma que la entropía de todo sistema aislado o permanece constante (en los procesos reversibles) o aumenta (en los procesos irreversibles). En cambio, los seres vivos como sistemas abiertos, intercambian energía con el exterior, son capaces de disminuir su entropía y crear estructuras altamente ordenadas absorbiendo energía del exterior lo cual implica un aumento de entropía externa (ORTUÑO ORTÍN, 1996). Así, la estabilidad de estos sistemas abiertos que operan lejos del equilibrio puede ser comprendida en términos de dos mecanismos organizadores básicos: la regulación (CAPRA, 1996) considerada como mecanismo para mantener un equilibrio interno en el animal y por tanto, un proceso homeostático.

Uno de los principales medios por los cuales el ambiente influye en los animales es a través del intercambio de energía. Un animal puede estar energéticamente en equilibrio con el ambiente, cuando la energía ganada por su cuerpo es igual a la energía liberada al entorno; pero si la energía ganada es superior a la perdida, el animal puede sobrecalentarse y morir, mientras que si la energía liberada al entorno es superior a la energía ganada de éste, el animal se enfriará y perecerá (PORTER; GATES, 1969).

En este sentido, la regulación de la temperatura en los animales, particularmente, en los lagartos es una temática que llama la atención por la complejidad que implica una explicación admisible. Un trabajo considerado como la base de la mayoría de estudios sobre biología termal en los lagartos es el desarrollado por Cowles y Bogert (1944). Estos dos autores demuestran que una creencia mantenida desde Aristóteles, que clasificaba a los animales en organismos de sangre fría y de sangre caliente, era una concepción errónea. Los lagartos pueden regular la temperatura de su cuerpo con considerable precisión y el rango en el que la temperatura es regulada es característico de cada especie. Desde 1940 se ha aceptado la ectotermia en lugar de la poiquilotermia (BICEGO et al., 2007) para señalar aquella condición de los animales cuyo calor corporal depende del ambiente, y la endotermia en lugar de la homeotermia (COWLES, 1962) para referirse a los animales que regulan su temperatura corporal con fuentes de energía internas.

En el caso de los organismos endotermos, como los mamíferos, la fuente principal de energía es interna, y se debe principalmente al alto metabolismo oxidativo. Así, la energía calórica liberada en el metabolismo, proceso mediante el cual los seres vivos convierten los alimentos en componentes celulares mediante una red altamente integrada de reacciones químicas (WOLFE, 1996), contribuye en el mantenimiento de una temperatura corporal, en general, constante.

Algo distinto ocurre en animales ectotermos como los lagartos cuya fuente de energía es externa debido a que la capacidad para generar energía mediante el metabolismo es insignificante. En estos reptiles, los procesos metabólicos no son 100\% eficientes y parte de la energía química se pierde como calor cuando el ATP es sintetizado e hidrolizado lo que hace que pocos empleen la producción de energía metabólica para acelerar el calentamiento y retardar el 
Daza-Pérez, E. P.; Pérez-Miranda, R.

enfriamiento o para mantener su temperatura corporal constante. Entonces, el intercambio de energía con el ambiente es más importante para determinar su temperatura corporal que la generada por procesos metabólicos (POUGH et al., 2003). En consecuencia, los lagartos termorregulan mediante procesos fisiológicos y principalmente comportamentales (Cuadro 1), para evitar temperaturas corporales extremadamente peligrosas y probablemente, realizar algún control sobre procesos metabólicos (HUEY; SLATKIN, 1976).

Cuadro 1. Mecanismos empleados por ectotermos para regular la temperatura corporal.

\begin{tabular}{|l|l|}
\hline \multicolumn{2}{|c|}{ Mecanismos } \\
\hline Comportamental & \multicolumn{1}{|c|}{ Fisiológico } \\
\hline Variación estacional de la temperatura corporal media & Piel \\
\hline Selección de microhabitat & Absorbancia \\
\hline Sol versus sombra & Producción de calor \\
\hline Uso de madrigueras & Metabolismo \\
\hline Escalamiento (subir alturas) & Actividad muscular \\
\hline Agrupamiento animal & Enfriamiento por evaporación \\
\hline Ajustes posturales & Pérdida cutánea \\
\hline Orientación al sol & Piel seca \\
\hline Orientación al viento & Piel húmeda \\
\hline Cambios morfológicos & Jadeo \\
\hline Elevarse fuera del sustrato & Salivación \\
\hline Conducción por el sustrato & Orina \\
\hline & Sistema cardiovascular \\
\hline & Control del flujo de la sangre \\
\hline & Superficie corporal \\
\hline
\end{tabular}

Fuente: tomado de Stevenson, 1995.

Dado que en el cuadro anterior sólo menciona algunas respuestas, en la figura 1 se explica el proceso de termorregulación en lagartos. Debido a que la energía generada en procesos metabólicos es baja y se disipa rápidamente en el entorno, el lagarto obtiene energía de fuentes externas mediante mecanismos biofísicos.

La energía solar que es tomada directamente o mediante, convección, radiación y/o conducción, puede ser perdida por evaporación y convección. Esa ganancia y esa pérdida de energía conducen a un estado de equilibrio energético que permite al lagarto llevar a cabo eficazmente sus procesos fisiológicos. Ahora, si ese equilibrio térmico es alterado y sobrepasa los rangos de tolerancia térmica para la especie, se generan respuestas comportamentales y fisiológicas contra el enfriamiento y el sobrecalentamiento generadas por el sistema nervioso 
Termorregulación de lagartos en la formación de profesores ...

(ver Cuadro 1). De acuerdo con Tattersall, Cadena y Skinner (2006) los mecanismos fisiológicos incluyen cambios en el ritmo cardíaco, ajustes circulatorios, termogénesis y, una forma poco explorada desde la perspectiva fisiológica, el enfriamiento mediante la respiración.

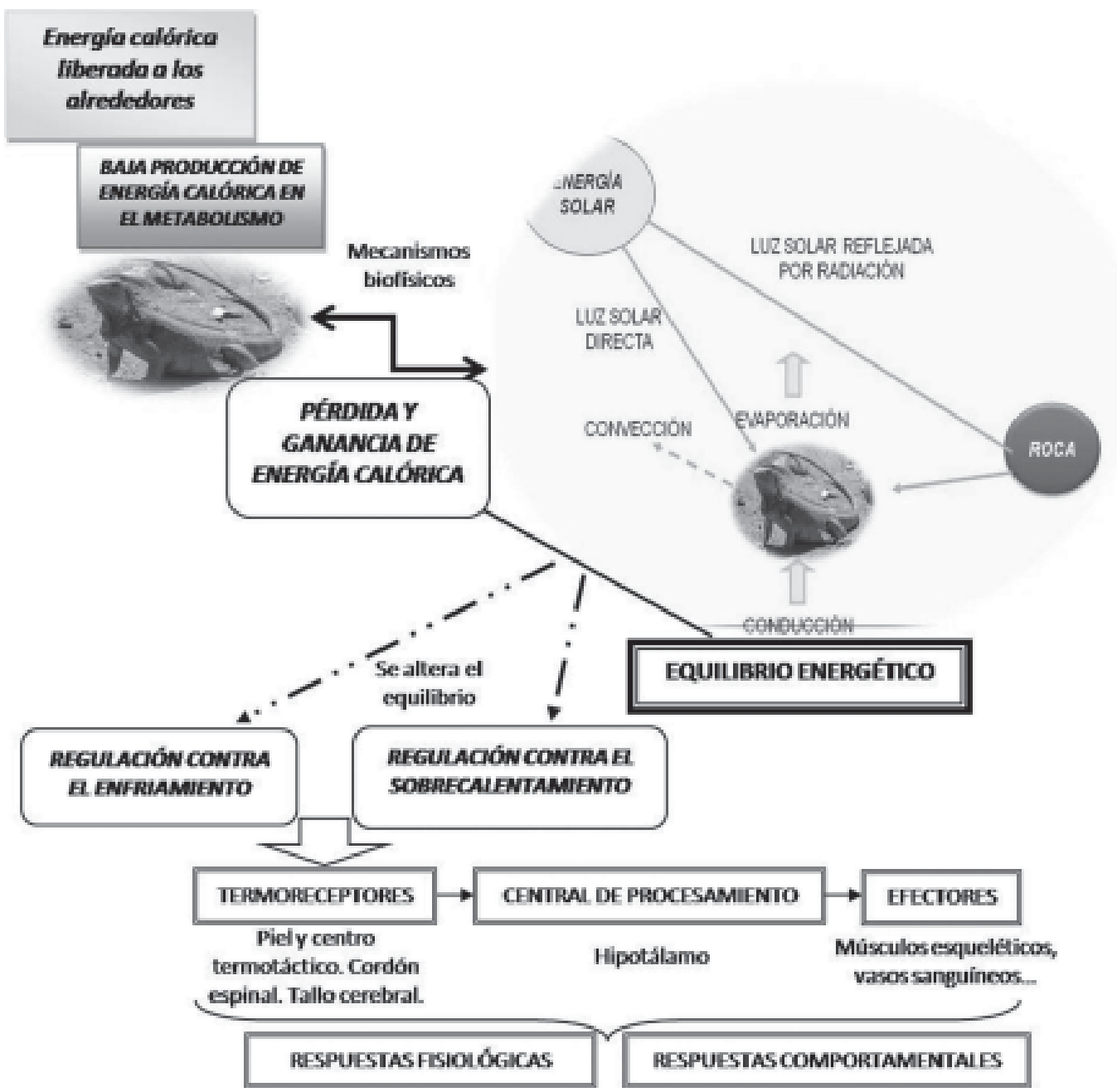

Figura 1. Mecanismo de regulación térmica en los lagartos. Adaptación de Pough et al., (2003). Se integran las propuestas de Barbour (1921) y Bicego et al. (2007). La temperatura corporal del animal resulta del balance entre la energía perdida y la energía ganada mediante diferentes caminos biofísicos. 
Daza-Pérez, E. P.; Pérez-Miranda, R.

\section{Termorregulación en lagartos y los modelos de profesores de ciencias en formación inicial}

A partir del modelo propuesto y lo discutido en el apartado anterior, se establecieron las categorías desde las cuales se analizaron los modelos elaborados por los profesores de ciencias naturales y educación ambiental, en formación inicial.

\section{Metodología}

El estudio se desarrolló con 24 profesores en formación inicial de la Licenciatura en Ciencias Naturales y Educación Ambiental de la Universidad Pedagógica y Tecnológica de Colombia en tres etapas que se resumen en la Figura 2.

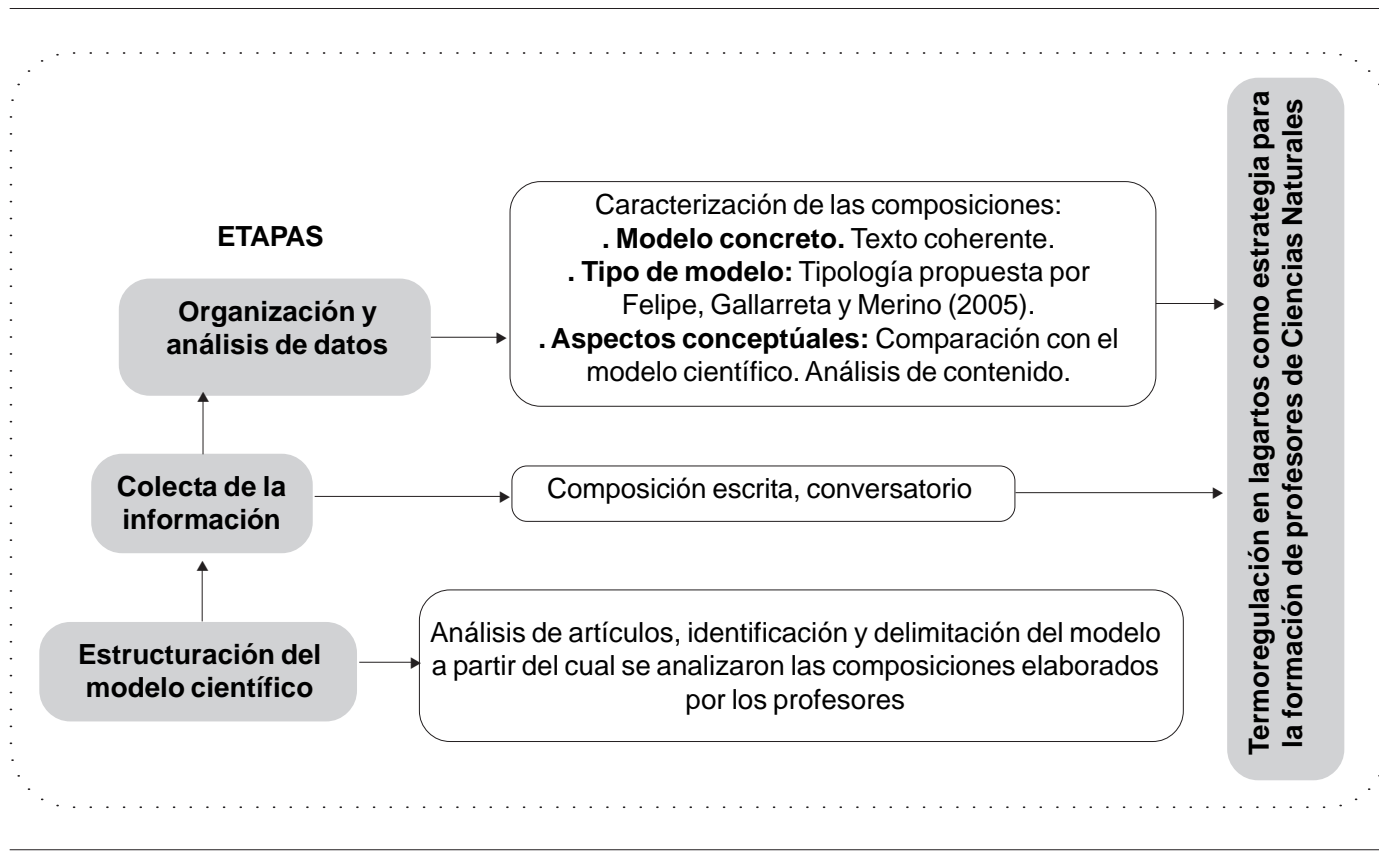

Figura 2. Metodología. Se indican las etapas y actividades desarrolladas en cada una de ellas. Se partió del análisis de los modelos científicos y como meta final argumentar mediante una propuesta teórica sobre el la importancia de hacer objeto de estudio en la formación de Profesores de Ciencias el modelo científico sobre la termorregulación de lagartos.

En la fase de recolección de la información, los profesores en formación elaboraron composiciones escritas en las que explicaban el mecanismo de regulación térmica de los vertebrados, centrándose en los lagartos. Además, resolvieron algunos interrogantes encaminados a ampliar la información obtenida en sus composiciones y que emplearan diversos recursos (gráficas, tablas, símbolos, analogías, ecuaciones, y otros) para explicar el proceso en cuestión. 
Termorregulación de lagartos en la formación de profesores ...

Con el objeto de clarificar algunas afirmaciones, gráficos y respuestas presentadas por los estudiantes, se realizaron entrevistas no estructuradas con cada estudiante y se les solicitó explicaciones más elaboradas y de mayor aceptabilidad a la pregunta ¿cómo regulaban la temperatura corporal los lagartos?

\section{Caracterización y análisis de los modelos}

La información colectada fue analizada de acuerdo con las siguientes categorías:

- Existencia de un modelo sobre la regulación térmica: Se analizó si los profesores en formación planteaban un texto con sentido en el que se explicaba el proceso de regulación de la temperatura corporal en los lagartos. Si se incluían diferentes tipos de respuestas a las variaciones en la temperatura ambiental o si por el contrario sólo se mencionaban afirmaciones aisladas.

- Tipo de modelo: Se empleó la tipología propuesta por Felipe, Gallarreta y Merino (2005). Se señalaron aquellos de sentido común y los que se corresponden con lo aceptado por la comunidad de especialistas.

- Los profesores en referencia emplearon otros recursos para expresar sus modelos respecto al tema en cuestión. Estas representaciones fueron clasificadas en modelos icónicos, analógicos o simbólicos: dibujos de animales, gráficas estadísticas, esquemas conceptuales, ecuaciones y otros. Estos modelos fueron analizados de acuerdo con los interrogantes que se plantearon en los aspectos conceptuales.

- Aspectos conceptuales: de las composiciones se tomaron frases o palabras que permitían establecer la aproximación que sobre regulación de la temperatura corporal tenían los profesores de ciencias naturales y educación ambiental en formación inicial y que son aceptadas científicamente y sobre la clasificación que se hace de los vertebrados de acuerdo con la termorregulación.

Esta caracterización se fundamentó con los siguientes interrogantes:

¿Se reconocen diferencias en los mecanismos termoregulatorios entre los diversos grupos de vertebrados? ¿En qué grupo de vertebrados se muestra un mayor conocimiento? En el caso de los lagartos, ¿Qué tipo de respuestas se mencionan cuando el animal se expone al sobrecalentamiento y al enfriamiento?, ¿Qué términos y/o afirmaciones empleados no son aceptados científicamente en relación con la termorregulación de los lagartos?, ¿Desde qué perspectiva (física, química o biología) se explica la termorregulación en lagartos?, ¿Los modelos incluyen aspectos de las diferentes ciencias de la naturaleza? ¿Sólo se limitan a explicaciones desde la perspectiva biológicas? Y, en general, ¿Cómo explican los profesores en referencia el proceso de regulación de la temperatura corporal en los lagartos?

\section{Resultados}

Tras analizar las composiciones se pudo identificar que los profesores en formación no han estructurado un modelo sobre el proceso de regulación de la temperatura en los lagartos, que corresponda con lo propuesto por la comunidad de especialistas. Sus explicaciones 
son teórico-descriptivas de sentido común y en algunas se evidencian vaguedades como el considerar que los sapos son reptiles: '[...] el lagartijo si podría sobrevivir en temperaturas elevadas puesto que su adaptación al calor es mejor y tiene métodos para combatir el calor. En cuanto al frío moriría porque es de sangre fría y busca es las zonas cálidas”. También se afirma que soportan altas temperaturas porque su piel permite conservar la humedad: "[...] su gruesa piel impide la pérdida desmesurada de agua puesto que es rígida y gruesa".

Los resultados indican que la temática en cuestión aún no ha sido objeto de trabajo en el aula como contenido curricular específico de la formación de los profesores de Ciencias Naturales y Educación Ambiental. Son escasas las manifestaciones en cuanto a la estructuración de modelos, por parte de estos profesores.

- Tipo de modelo y aspectos conceptúales: de acuerdo con la tipología adoptada, la mayoría de las composiciones (22) se clasificaron como modelos teóricos funcionales, un profesor elaboró un modelo funcional de tipo analógico:

Los animales se desarrollan en un medio ambiente en el cual están sujetos a la temperatura de su entorno ya que cuando bay una pérdida calórica ocurre un proceso similar a lo que se explica desde la segunda ley de la termodinámica que dice que el calor es transferido desde un cuerpo más caliente a un cuerpo más frío.

Sólo siete (7) profesores elaboraron textos con sentido, en los que existe conexión entre las ideas y procuran presentar un modelo estructurado sobre la regulación térmica.

Las representaciones gráficas corresponden a modelos icónicos. Los mismos fueron clasificados como esquemas conceptúales, dibujos, gráficas estadísticas y otras gráficas como lo explicita la Figura 3.

Aunque el tema no es explícito en los programas de la Educación Básica ni en los de Educación Media (Estándares básicos de competencia en Ciencias) como tampoco en la Educación Superior, en este caso los que corresponden al programa de formación inicial de profesores de ciencias naturales y educación ambiental, el currículo de la misma incluye asignaturas como fisiología, física, biofísica, biología animal II química general y bioquímica en las que se asume un trabajo sobre conceptos que permiten comprender aspectos de bioenergética, mecanismos físicos de pérdida y ganancia de energía en animales y fundamentalmente, términos como ectotermia y endotermia. Además, se encontró que en diecisiete (17) profesores aún predomina el modelo Aristotélico en el que se reconoce que los animales pueden clasificarse en organismos de sangre fría y de sangre caliente, y que los lagartos no han desarrollado rangos finitos de tolerancia térmica según la especie. En estos profesores tampoco hay, como se muestra en las Figuras 4, 5 e 6, consenso sobre la terminología empleada para referirse a la biologia termal de los diferentes grupos de animales. Usan términos como animales con regulación externa e interna, poiquilotermos y endotermos, heterotermos y se sangre fría entre otros.

Cabe aclarar que aunque se acogió la ectotermia y endotermia como condiciones relacionadas con mecanismos de obtención de energía y regulación de la temperatura corporal, existen especies que ocupan posiciones intermedias. Este aspecto tampoco es abordado por los profesores de ciencias naturales y educación ambiental en formación inicial en sus composiciones en el análisis del fenómeno trabajado. 
Termorregulación de lagartos en la formación de profesores ...

\begin{tabular}{|c|c|c|c|}
\hline $\begin{array}{l}\text { Tipo de } \\
\text { modelo }\end{array}$ & Características & Explicaciones centrales & $\begin{array}{c}\text { Aspectos } \\
\text { no aceptados }\end{array}$ \\
\hline 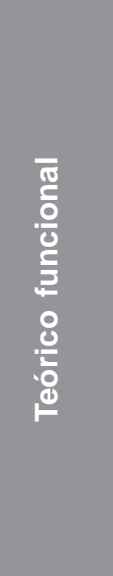 & $\begin{array}{l}\text { Frases aisladas, se } \\
\text { mencionan algunos } \\
\text { mecanismos. } \\
\text { Las explicaciones se } \\
\text { basan principalmente } \\
\text { en aspectos } \\
\text { fisiológicos y } \\
\text { comportamentales } \\
\text { Citan términos como } \\
\text { energía, calor y } \\
\text { conducción del calor } \\
\text { pero no se explicita un } \\
\text { tratamiento que } \\
\text { corresponda con esas } \\
\text { perspectivas. }\end{array}$ & $\begin{array}{l}\text { Los lagartos se adaptan fácilmente } \\
\text { a temperaturas elevadas } \\
\text {... los animales de sangre fría están } \\
\text { adaptados a ambientes húmedos y } \\
\text { al pasar a climas cálidos se } \\
\text { sofocan... } \\
\text { Los lagartos han desarrollado } \\
\text { adaptaciones morfológicas para } \\
\text { termorregular. } \\
\text { Desarrollan respuestas fisiológicas } \\
\text { (pueden sufrir de hipotermia) y } \\
\text { comportamentales (buscar sombra, } \\
\text { cambiar de postura) }\end{array}$ & $\begin{array}{l}\text { Predomina el modelo } \\
\text { Aristotélico, organismos } \\
\text { de sangre fría y de } \\
\text { sangre caliente ... los } \\
\text { lagartos no han } \\
\text { desarrollado rangos } \\
\text { finitos de tolerancia } \\
\text { térmica. } \\
\text { Carencia de un consenso } \\
\text { en cuanto a la } \\
\text { terminologíca } \\
\text { (poiquilotermos, } \\
\text { regulación interna, } \\
\text { sangre fría...). }\end{array}$ \\
\hline 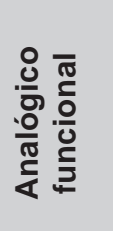 & $\begin{array}{l}\text { Comparación con la } \\
\text { segunda ley de la } \\
\text { termodinámica }\end{array}$ & $\begin{array}{l}\text { Los animales están sujetos a la } \\
\text { temperatura de su entorno } \\
\text { entonces, una diferencia de } \\
\text { temperatura genera una pérdida } \\
\text { de calor de uno de los cuerpos. }\end{array}$ & $\begin{array}{l}\text { Proceso similar a lo que } \\
\text { se explica desde la } \\
\text { segunda ley de la } \\
\text { termodinámica... el calor } \\
\text { es transferido desde un } \\
\text { cuerpo más caliente a un } \\
\text { cuerpo más frio. }\end{array}$ \\
\hline \multirow{4}{*}{ 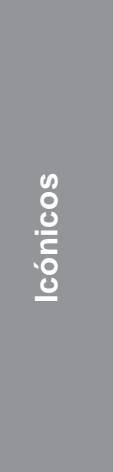 } & $\begin{array}{l}\text { Esquemas } \\
\text { conceptúales: } \\
\text { esquemas con } \\
\text { información teórica y } \\
\text { dibujos de animales. }\end{array}$ & $\begin{array}{c}\text { Indica el tipo de respuesta y el órgano } \\
\text { involucrado. }\end{array}$ & $\begin{array}{l}\text { A diferencia de los demás } \\
\text { vertebrados, el lagarto sí } \\
\text { se adapta a altas } \\
\text { temperaturas. }\end{array}$ \\
\hline & $\begin{array}{l}\text { Diburjos de lagartos: } \\
\text { (Fig. 4) }\end{array}$ & Indica el tipo de respuesta. & \\
\hline & $\begin{array}{c}\text { Gráficas estadísticas: } \\
\text { (Fig. 5) }\end{array}$ & $\begin{array}{l}\text { Intenta mostrar qué vertebrado podría } \\
\text { adaptarse a altar o bajas temperaturas }\end{array}$ & $\begin{array}{l}\text { El lagarto es el animal } \\
\text { que mejor se adapta a los } \\
\text { cambios de temperatura }\end{array}$ \\
\hline & Otras gráficas: (Fig. 6) & $\begin{array}{l}\text { Relaciona las adaptaciones y en la } \\
\text { evolución de las especies. }\end{array}$ & \\
\hline
\end{tabular}

Figura 3. Modelos propuestos por los profesores en formación para explicar el mecanismo de termorregulación en los lagartos.

- Aspectos de física, química y biología empleados en las explicaciones: Las composiciones se basan principalmente en aspectos fisiológicos y biológicos como el comportamiento. Se citan términos como energía, calor, metabolismo y conducción del calor pero no se explicita un tratamiento que corresponda con esas perspectivas. Dos de estos profesores incluyen algunos tratamientos químicos o físicos. Uno de ellos indica que en el mecanismo de regulación de la temperatura corporal están involucrados procesos químicos y físicos: 
Daza-Pérez, E. P.; Pérez-Miranda, R.

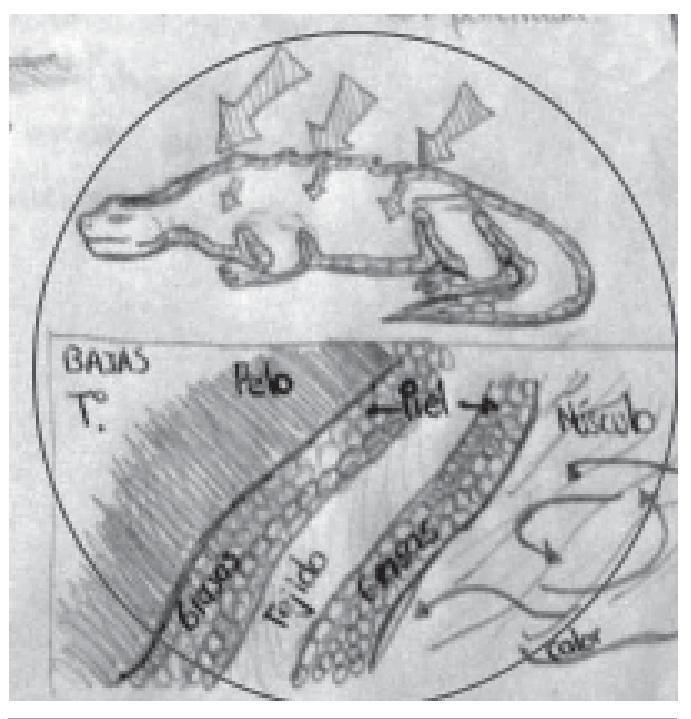

Figura 4. Dibujos de lagartos. El modelo es una aproximación a lo aceptado por la comunidad científica y al modelo del cilindro propuesto por Porter y Gates (1969), y adaptado por O'Connor (1999), en el que se considera un intercambio de calor entre las diversas capas (conchas, según el artículo) que conforman el animal.

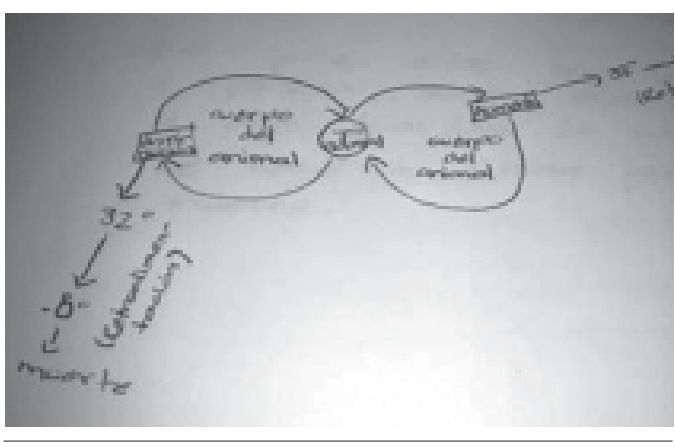

Figura 5. El modelo se centra en las adaptaciones y en la evolución de las especies.

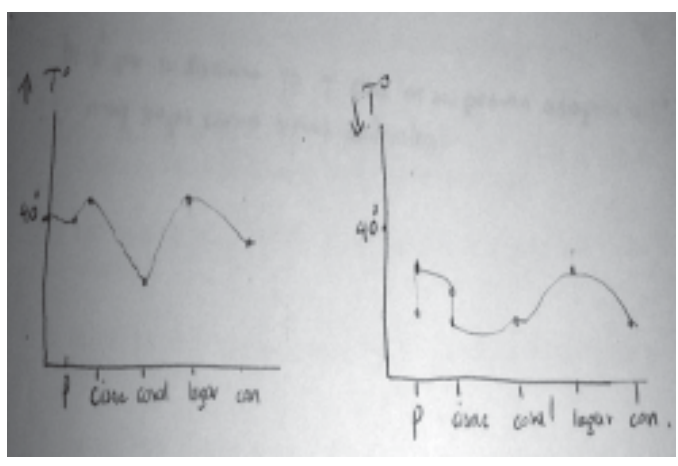

Figura 6. Gráficas estadísticas. Respuesta de los vertebrados a un incremento o un descenso de la temperatura, en términos de adaptación. Según esto, el lagarto es el animal que mejor se adapta a los cambios de temperatura.

[...] el metabolismo, la nutrición, la respiración y la circulación son procesos bioquimicos que contribuyen a atender las necesidades térmicas de los animales. Por ejemplo, se obtiene energía química y se produce energía calórica. A partir de la energía cinética, produce energía calórica. Existen animales de sangre fría en climas calientes esto ayuda a controlar la temperatura adecuada para la supervivencia. Un cambio físico de un animal se puede reflejar en la abundancia de pelaje.

Como puede notarse, se apela a lo descriptivo de este fenómeno. No se acude al tratamiento químico que se espera para este caso. Tampoco hay precisión en las leyes termodinámicas. Hace una analogía entre la termodinámica y la regulación térmica, un modelo que también ha sido propuesto por la comunidad de especialistas. 
Termorregulación de lagartos en la formación de profesores ...

Los animales se desarrollan en un medio ambiente en el cual están sujetos a la temperatura de su entorno ya que cuando hay una pérdida calórica ocurre un proceso similar a lo que se explica desde la segunda ley de la termodinámica que dice que el calor es transferido desde un cuerpo más caliente a un cuerpo más frío.

En este caso como en el anterior, se limita a lo narrativo del fenómeno influenciado por la carencia de precisión en el tratamiento termodinámico. Obsérvese que a pesar de mencionar algunos elementos de la física, no hay una explicación concreta desde esa perspectiva. Precísese que el análisis de los resultados arrojados muestran un fuerte sesgo a la perspectiva biológica fundamentados en lo descriptivo, y algunos casos relacionados con explicaciones fisiológicas y comportamentales.

Tanto en las composiciones como en las gráficas se evidencian vaguedades. Los profesores en formación inicial consideran que los lagartos habitan en climas cálidos porque su condición de organismos ectotermos le permite soportar altas temperaturas, clasifican a los lagartos como organismos de sangre fría y en ocasiones a los peces como organismos de sangre caliente. También se proponen otras explicaciones que no se aproximan a lo propuesto por la comunidad científica como el considerar el sapo como un reptil.

[...] los reptiles como el lagarto y el sapo que son de sangre fría, en temperaturas muy altas se sitúan paralelos al sol y contraen su caja torácica para recibir menos irradiación. Algunos se refugian debajo de lodo o de arena. En climas fríos estos cambian la coloración de su piel a un color más claro para regular el calor.

Pese a ello, algunas afirmaciones hechas en las composiciones como el reconocer en los lagartos varias respuestas comportamentales son válidas, y deben ser tenidas en cuenta a la hora de implementar una estrategia para el trabajo acerca de este fenómeno.

Con base en esto resultados, se puede afirmar que es fundamental involucrar en la formación de profesores de Ciencias Naturales y Educación Ambiental los modelos formulados sobre termorregulación en lagartos porque es una temática que permitiría a los profesores de ciencias naturales y educación ambiental trabajar mecanismos de obtención de energía en los seres vivos, establecer relaciones entre el clima y las adaptaciones y analizar e identificar criterios tenidos en cuenta para clasificar los organismos en diversos grupos taxonómicos. Así como la integración de modelos explicativos sobre fenómenos que como termorregulación pueden ser explicados desde distintos campos del conocimiento (física, química, biología, matemáticas, entre otros).

Con la convicción de que el docente ha de estar formado bajo los principios epistemológicos e históricos y conocer el modelo que será objeto de aprendizaje por parte de sus estudiantes es necesario que los profesores reconozcan la termorregulación como un proceso de homeostasis puesto que este último, es considerado como uno de los conceptos que ha influenciado en la historia de la biología (MAYR, 1998), provee el armazón conceptual dentro de la cual se interpreta un amplio rango de datos fisiológicos y permite comprender aspectos fundamentales sobre el funcionamiento de los seres vivos.

Ante lo evidenciado por los resultados obtenidos y analizados, se optó por explorar algunas razones por las cuales se producen esos resultados cuando se esperaban otros. Se 
procedió a revisar las temáticas propuestas en los programas curriculares de las distintas asignaturas (biología animal, fisiología, biofísica, bioquímica y termodinámica) que se piensa tienen relación con el fenómeno trabajado, la termorregulación. En esta somera revisión se observó que se proponen contenidos que favorecen una formación, que permite a los futuros profesores construir explicaciones sobre la termorregulación desde una perspectiva integradora.

El trabajar en el aula termobiología, biología de reptiles, termodinámica, calor y energía, metabolismo y termodinámica entre otros, proporciona los fundamentos necesarios para la estructuración de un modelo que integre la química, biología y física, ciencias que han de ser fundamentales en la formación de profesores de Ciencias Naturales. Cabe preguntarse entonces, por la forma como se orientan las diferentes asignaturas, el proceso de formación de estos profesores en cuanto al conocimiento de sus campos de formación (biología, química, física y educación ambiental) y al tratamiento de los contenidos en el aula, ¿'Realmente se hacen objeto de trabajo en el aula los contenidos propuestos en los planes curriculares?, ¿Cómo se trabajan?, ¿Por qué estos profesores de ciencias naturales y educción ambiental en formación inicial no relacionan esos contenidos? ¿Qué otros fenómenos se considerarían como de la misma categoría de complejidad para su trabajo en el aula que conforman los contenidos curriculares en esa formación?

\section{Termorregulación en lagartos, un modelo básico en la formación de Licenciados en Ciencias Naturales}

Teniendo en cuenta los resultados, la inquietud que se manifiesta, es la posibilidad de superar estas situaciones problema en la formación de los profesores de Ciencias Naturales y Educación Ambiental, no sólo en cuanto a termorregulación se refiere sino en cuanto a aquellas temáticas que por alguna razón no se hacen objeto de trabajo en el aula, ya sea por no ser explícita en los programas de las asignaturas que conforman el plan de estudios o porque no sean del interés del grupo aula en su momento, pero que dados los desarrollos tecnológicos y de las mismas ciencias podrían presentarse y que los profesores necesariamente han de abordarlo. Se presenta así una propuesta sobre cómo podría incluirse en la formación de esos profesores la termorregulación en lagartos que se asumirá como un problema didáctico que sea objeto de investigación (Figura 7).

Considerando que los profesores a menudo propician en los estudiantes la formación de modelos de los procesos vitales como entidades aisladas, capaces de ser realizadas de manera independiente (TAMAYO ALZATE, 2001; GÓMEZ GALINDO, 2005), y que para muchos estudiantes las relaciones entre seres vivos y células existen sólo en seres humanos (PEDRANCINI et al., 2007), es fundamental que se haga objeto de trabajo en el aula una propuesta que permita una comprensión del universo biológico organizado, a partir del tipo de las interacciones que se dan entre los mismos. En ese sentido, se propone estudiar fenómenos físicos, químicos y biológicos y problemáticas ambientales desde la termorregulación en lagartos sin distanciarse de las cuestiones histórico - epistemológicas de cada disciplina científica.

Desde el punto de vista histórico-epistemológico es necesario comprender cómo se han constituido la biología, la física y la química como ciencias de la naturaleza, cuáles son sus características y por qué el ser vivo reconocido como un sistema, no puede ser reducido a las 


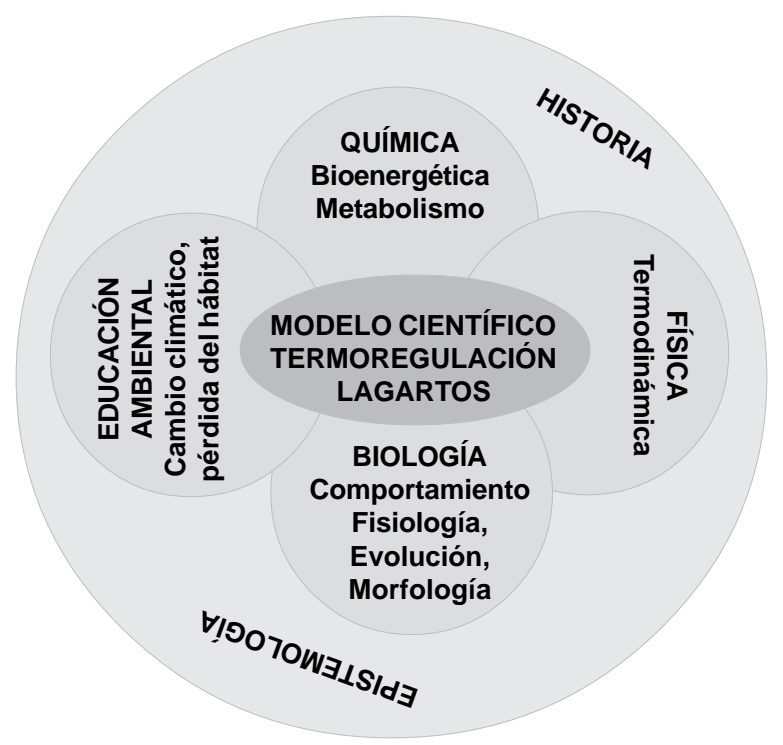

Figura 7. Temáticas generales en las que puede integrarse el modelo científico de termorregulación en lagartos. Se indica una estrecha relación entre las áreas y temas propuestos, un trabajo interdisciplinar centrado en la termorregulación en lagartos y basado en la historia y epistemología de cada disciplina.

leyes de la física o la química aunque que varios fenómenos logran ser interpretados desde ellas. Con este componente, los profesores habrían de conocer y trabajar, en la reconstrucción histórica de la termorregulación en lagartos, el concepto de calor, energía y las leyes de la termodinámica.

Mediante un trabajo interdisciplinar basado en la historia y epistemologia, se integraría el estudio de los aspectos físicos, biológicos (fisiológicos, etológicos) y químicos (bioquímica) desde los cuales puede interpretarse el fenómeno en cuestión (Figura 7). El componente de la educación ambiental podrá ser abordado desde las problemáticas que actualmente enfrenta la diversidad biológica, como la pérdida del hábitat o el efecto del cambio climático sobre este grupo de vertebrados en específico o sobre la herpetofauna, en general.

Entonces, ¿Qué estrategia seguir para lograr esta integración? El desarrollo de una estrategia de formación que responda a ese propósito requiere de un trabajo interdisciplinar. Desde cualquiera de las asignaturas puede ser propuesto, por ejemplo, la construcción de modelos (JUSTI, 2006) que expliquen el proceso apelando a las explicaciones que se han dado desde las ciencias de la naturaleza. No obstante, esta es la cuestión que nos ocupa sobre y sobre la cual pretendemos desarrollar una investigación. 
Daza-Pérez, E. P.; Pérez-Miranda, R.

\section{A manera de conclusión}

Los resultados del presente estudio constituyen la base para el desarrollo de investigaciones sobre el tratamiento en el aula y desarrollo histórico - epistemológico de la temática (DAZA-PÉREZ, 2008) presenta una aproximación en este campo. Teniendo en cuenta que el proceso ha sido objeto de trabajo por especialistas en herpetología y fisiología, y no se ha incluido como tema de investigación por parte de didactas de la ciencia, cabe preguntarse ¿Hasta dónde otros fenómenos de estas mismas características se dejan de lado en la formación de profesores de ciencias?

Se espera que esta propuesta se constituya en una generalización para otros fenómenos no trabajados en la formación de profesores de ciencias naturales y educación ambiental como objeto de conocimiento para analizar las concepciones que se poseen en pro de una versión admisible sobre la ciencia y los conceptos científicos que hacen parte de la formación de estos profesores.

\section{Referencias}

ADÚRIZ-BRAVO, A.: IZQUIERDO, M.; ESTANY, A. Una propuesta para estructurar la enseñanza de la filosofía de la ciencia para el profesorado de ciencias en formación.

Enseñanza de las Ciencias, Barcelona, v. 20, n. 3, p. 465-476, 2002.

BARBOUR, H. The heat-regulating mechanism of the body. Physiological Reviews, Bethesda, v. 1, n. 2, p. 295-326, 1921.

BELTRÁN VILLAMIZAR, Y.; QUIJANO HERNÁNDEZ, M. H.; VILLAMIZAR ACEVEDO, G. A. Concepciones y prácticas pedagógicas de los profesores que enseñan ciencias naturales y ciencias humanas en programas de ingeniería de dos universidades colombianas. Studiositas, Bogota, v. 3, n. 1, p. 41- 45, 2008.

BICEGO, K.; BARROS, R.; BRANCO, L. Physiology of temperature regulation: comparative aspects. Comparative Biochemistry and Physiology, Part A: Molecular \& Integrative Physiology, Philadelphia, v. 147, n. 3, p. 616-639, 2007.

CAPRA, F. La trama de la vida. Una perspectiva de los sistemas vivos. Barcelona: Editorial Anagrama, 1998.

COLOMBIA. Ministerio de Educación Nacional. Formar en ciencias: jel desafío! lo que necesitamos saber y saber hacer. Bogotá, 2004. (Estándares básicos de competencias en ciencias naturales y ciencias sociales. Serie guías, n. 7).

COWLES, R.; BOGERT, C. A preliminary study of thermal requirements of desert reptiles. Bulletin of the American Museum of Natural History, New York, v. 83, n. 5, p. 265-296, 1944. 
Termorregulación de lagartos en la formación de profesores ...

COWLES, R. Semantics in biothermal studies. Science, New York, v. 135, n. 3504, p. 670, 1962.

DAZA-PÉREZ, E. Termorregulación en lagartos: modelos elaborados por profesores de ciencias en formación inicial. 2008. 139f. Tesis (Maestría en docencia de la Química) Facultad de Ciencia y Tecnología, Universidad Pedagógica Nacional, Bogotá, 2008.

ECKERT, R. et al. Animal physiology: mechanisms and adaptations. 5. ed. New York: Freeman, 2001.

FELIPE, A.; GALLARRETA, S.; MERINO, G. La modelización en la enseñanza de la biología del desarrollo. Revista Electrónica de Enseñanza de las Ciencias, v. 4, n. 3, 2005. Disponible en: <http://www.saum.uvigo.es/reec/volumenes/volumen4/ ART5_Vol4_N3.pdf>

GÓMEZ GALINDO, A. A. La construcción de un modelo de ser vivo en la escuela primaria: una visión escalar. 2005. 463f. Tesis (Doctorado en Ciencias de la Educación) Departament de Didactica de la Matematica i de les Ciencies Experimentals, Universitat Autonoma de Barcelona, Bellaterra, 2005.

GONZALÉZ, E. et al. Cinco ejes para la discusión sobre la formación inicial y la capacitación de los docentes de ciencias. Revista de Enseñanza de la Física, Córdoba, v. 9, n. 2, p. 75-83, 1996.

HUEY, R.; SLATKIN, M. Cost and benefits of lizard thermoregulation. The Quarterly Review of Biology, New York, v. 51, n. 3, p. 363-384, 1976.

JUSTI, R. La enseñanza basada en la elaboración de modelos. Enseñanza de las Ciencias, Barcelona, v. 24, n. 2, p. 173-184, 2006.

MAYR, E. Así es la biología. Madrid: Debate, 1998.

MAYR, E. The autonomy of biology. Ludus Vitalis: Revista de Filosofía de las Ciencias de la Vida, México, v. 12, n. 21, p. 15-28, 2004.

MELLADO, V. Concepciones y prácticas de aula de profesores de ciencias en formación inicial de primaria y secundaria. Enseñanza de las Ciencias, Barcelona, v. 14, n. 3, p. 289-302, 1996.

O'CONNOR, M. Physiological and ecological implications of a simple model of heating and cooling in reptiles. Journal of Thermal Biology, Oxford, GB, v. 24, n. 2, p. 113-136, 1999.

ORTUÑO ORTÍN, M. Física: para biología, medicina, veterinaria y farmacia. Barcelona: Crítica, 1996.

PEDRANCINI, V. et al. Ensino e aprendizagem de Biologia no ensino médio e a apropriação do saber científico e biotecnológico. Revista Electrónica de Enseñanza de las Ciencias, v. 6, n. 2, p. 299-309, 2007. Disponible en: <http://www.saum.uvigo.es/reec/ volumenes/volumen6/ART5_Vol6_N2.pdf> 
Daza-Pérez, E. P.; Pérez-Miranda, R.

PORTER, W.; GATES, D. Thermodynamic equilibria of animals with environment.

Ecological Monographs, Lawrence, v. 39, n. 3, p. 227-244, 1969.

POUGH, H. et al. Herpetology. 3. ed. New Jersey: Prentice Hall, 2003.

STEVENSON, R. The relative importance of behavioral and physiological adjustments controlling body temperature in terrestrial ectotherms. The American Naturalist, Chicago, v. 126, n. 3, p. 362-386, 1985.

TAMAYO ALZATE, O. E. Evolución conceptual desde una perspectiva multidimensional: aplicación al concepto de respiración. 2001. 328f. Tesis (Doctorado en Ciencias de la Educación) - Escuela de Doctorado y Formación Continuada, Universidad Autonoma de Barcelona, Barcelona, 2001.

TATTERSALL G. J.; CADENA, V.; SKINNER, M. C. Respiratory cooling and thermoregulatory coupling in reptiles. Respiratory Physiology \& Neurobiology, Oxford, GB, v. 154, n. 1-2, p. 302-18, 2006.

WOLFE, D. Química general, orgánica y biológica. México: McGraw Hill Interamericana, 1996.

Artigo recebido em 22/07/2010. Aceito em 14/12/2010. 